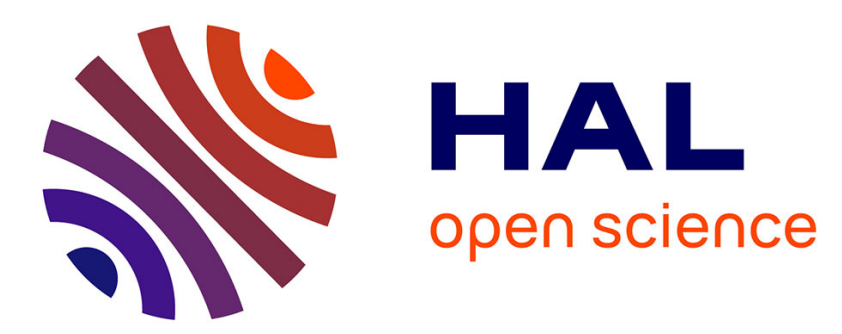

\title{
Naigeon lecteur de Diderot dans le dictionnaire de Philosophie ancienne et moderne de l'Encyclopédie méthodique
}

Claire Fauvergue

\section{- To cite this version:}

Claire Fauvergue. Naigeon lecteur de Diderot dans le dictionnaire de Philosophie ancienne et moderne de l'Encyclopédie méthodique. Recherches sur Diderot et sur l'Encyclopédie, 2015, 50, pp.105-119. $10.4000 /$ rde.5289 . hal-03241626

\section{HAL Id: hal-03241626 \\ https://hal.science/hal-03241626}

Submitted on 28 May 2021

HAL is a multi-disciplinary open access archive for the deposit and dissemination of scientific research documents, whether they are published or not. The documents may come from teaching and research institutions in France or abroad, or from public or private research centers.
L'archive ouverte pluridisciplinaire HAL, est destinée au dépôt et à la diffusion de documents scientifiques de niveau recherche, publiés ou non, émanant des établissements d'enseignement et de recherche français ou étrangers, des laboratoires publics ou privés. 


\section{Recherches sur Diderot et sur l'Encyclopédie}

$50 \mid 2015$

Varia

Naigeon lecteur de Diderot dans le dictionnaire de Philosophie ancienne et moderne de l'Encyclopédie méthodique

Naigeon's Reading of Diderot in the Dictionary of Ancient and Modern

Philosophy, Encyclopédie méthodique

Claire Fauvergue

\section{OpenEdition}

Journals

Édition électronique

URL : http://journals.openedition.org/rde/5289

DOI : $10.4000 /$ rde. 5289

ISSN : 1955-2416

Éditeur

Société Diderot

Édition imprimée

Date de publication : 25 novembre 2015

Pagination : 105-119

ISBN : 978-2-9520898-8-3

ISSN : $0769-0886$

\section{Référence électronique}

Claire Fauvergue, « Naigeon lecteur de Diderot dans le dictionnaire de Philosophie ancienne et moderne de l'Encyclopédie méthodique », Recherches sur Diderot et sur l'Encyclopédie [En ligne], 50 | 2015, mis en ligne le 25 novembre 2017, consulté le 04 janvier 2020. URL : http://journals.openedition.org/rde/5289 ; DOI : 10.4000/rde.5289 


\section{Claire FAUVERGUE}

\section{Naigeon lecteur de Diderot dans le dictionnaire de Philosophie ancienne et moderne de l'Encyclopédie méthodique}

Pour composer les trois volumes du dictionnaire de Philosophie ancienne et moderne pour l'Encyclopédie méthodique, Naigeon s'est appuyé sur des textes de Diderot, en particulier les articles de philosophie rédigés pour l'Encyclopédie. Dans Diderot et l'Encyclopédie, Jacques Proust a relevé les problèmes relatifs à l'établissement du texte diderotien dans l'Encyclopédie méthodique. Comme il en fait la remarque, « le texte de l'Encyclopédie méthodique est, à quelques variantes près, le texte même de l'Encyclopédie ". Bien qu'elles restent peu nombreuses, ces variantes manifestent chez Naigeon la volonté de rétablir le texte de la contribution de Diderot, en tenant compte des corrections manuscrites apportées par ce dernier à la première édition de l'Encyclopédie largement censurée. Naigeon entend également restituer dans l'Encyclopédie méthodique la pensée de Diderot ou encore, comme l'observe Jacques Proust, présenter Diderot « sous son vrai jour $\rangle^{1}$. Les commentaires de Naigeon accompagnant les articles de Diderot mettent en évidence la relation que ces articles entretiennent avec le projet philosophique diderotien. On peut, par conséquent, envisager l'attitude de Naigeon à l'égard des textes de Diderot en fonction de deux exigences : la restitution des contributions de Diderot à l'Encyclopédie et la reconstitution de son projet philosophique.

Dans son Mémoire sur Naigeon, Damiron attirait déjà l'attention sur les notes rédigées dans l'Encyclopédie méthodique par Naigeon, car « c'est là, le plus souvent, qu'il [Naigeon] marque le mieux son senti-

1. J. Proust, Diderot et l'Encyclopédie, 2e éd., Paris, Albin Michel, 1995, p. 146. 
ment $\rangle^{2}$. Damiron passe également en revue les articles dont Naigeon est l'auteur et où il affiche son athéisme. C'est au cours de l'analyse de l'article FATALISME ET FATALITÉ DES STOÏCIENS que Damiron relève l'emprunt que Naigeon fait à Diderot et s'exclame : " On reconnaîtra sans peine ici, et jusque dans ses termes mêmes, la pensée de Diderot, telle qu'il l'exprime dans une lettre que j'ai citée en son lieu ; c'est comme une leçon que le disciple répète d'après le maître [...] $»^{3}$. Naigeon reproduit en effet, dans l'article FATALISME, plusieurs passages de la Lettre à Landois ${ }^{4}$. Il faudrait préciser néanmoins qu'à ces passages viennent s'ajouter des extraits de l'article LIBERTÉs de l'Encyclopédie attribué en partie à Naigeon. La lecture à laquelle procède ce dernier dans l'article FATALISME suppose, par conséquent, tout un travail de lecture mais aussi de réécriture de textes dont certains seulement peuvent être attribués avec certitude à Diderot.

Certes, le projet de Naigeon dans le dictionnaire de Philosophie ancienne et moderne est bien de présenter Diderot sous son vrai jour, mais on peut se demander si cela passe exclusivement par la restitution du texte diderotien. En effet, dans le passage de l'article FATALISME qui a retenu l'attention des commentateurs, on observe que Naigeon présente la thèse soutenue par Diderot dans la Lettre à Landois et par lui-même dans l'article LIBERTÉ de l'Encyclopédie en renvoyant aux écrits de plusieurs philosophes parmi lesquels figurent Fontenelle, Bayle et Leibniz 6 . Or ces derniers renvois n'apparaissent pas dans le texte diderotien et leur étude s'avère essentielle afin de saisir la lecture de Diderot à laquelle procède Naigeon. De tels renvois prennent parfois dans l'Encyclopédie méthodique une forme nouvelle, celle de la citation d'extraits insérés dans le corps de l'article ou dans les notes. Ce premier constat nous amène à reconsidérer l'attitude de Naigeon à l'égard de Diderot. Naigeon ne se contente pas d'augmenter le volume des matériaux utiles à la constitution d'une histoire de la philosophie,

2. P. Damiron, Mémoire sur Naigeon [...] lu à l'Académie des sciences morales et politiques, Paris, A. Durand, 1857, p. 56.

3. Mémoire sur Naigeon, o.c., p. 53-54 ; Naigeon, Encyclopédie méthodique, Philosophie ancienne et moderne, article FATALISME ET FATALITÉ DES STOÏCIENS (Histoire de la philosophie ancienne et moderne), t. II, p. 408-409; l'Encyclopédie méthodique sera désormais citée $E M$.

4. Diderot, Lettre à Landois, DPV, IX, p. 257-258. Sur la question de l'emprunt de Naigeon à Diderot, voir H. Dieckmann, Inventaire du fonds Vandeul et inédits de Diderot, Genève, Droz, 1951, p. 148.

5. Naigeon, article LIBERTÉ (Morale), Enc., IX, 463.

6. Article FATALISME, EM, Philosophie ancienne et moderne, t. II, p. 409. Il s'agit du Traité de la liberté de Fontenelle, de l'article RORARIUS du Dictionnaire historique et critique de Bayle et des Essais de Théodicée de Leibniz. 
il prend la liberté d'insérer de nouveaux renvois dans le texte des articles de Diderot et d'en renouveler l'usage. Il développe ainsi une lecture originale de l'œuvre diderotienne en matière d'histoire de la philosophie tout en inscrivant celle-ci dans le nouveau contexte de l'Encyclopédie méthodique.

L'Encyclopédie méthodique, à la différence de l'Encyclopédie de Diderot et de D'Alembert, adopte une division des connaissances par ordre de matières. Les renvois internes à l'ouvrage encyclopédique sont remplacés par le vocabulaire, de telle sorte qu'au terme de la publication le lecteur devra consulter le « Vocabulaire encyclopédique $\gg^{7}$ prévu par Panckoucke, ce vocabulaire tenant lieu de table générale pour l'ouvrage entier. Ainsi conçu, le « Vocabulaire encyclopédique » remplace dans l'Encyclopédie méthodique les renvois de choses et de mots de la première Encyclopédie. Toutefois, le dictionnaire de Philosophie ancienne et moderne se distingue par la présence de nombreux renvois. Ainsi, leur insertion nous intéresse au même titre que les additions et les notes éditoriales : ces différents éléments sont l'expression de la lecture, par Naigeon, des articles dont Diderot est l'auteur dans l'Encyclopédie. On ne saurait par conséquent aborder le dictionnaire de Philosophie ancienne et moderne sans tenir compte de l'exploitation que Naigeon fait des renvois en éditant le texte diderotien. L'étude de ce nouvel emploi des renvois permettra notamment de saisir l'évolution que représente l'Encyclopédie méthodique par rapport à l'Encyclopédie de Diderot et D'Alembert en matière d'historiographie de la philosophie.

Diderot renouvelle l'éclectisme introduit par J. Brucker comme une notion critique s'appliquant à l'histoire de la philosophie en énonçant qu'aucun principe digne d'entrer dans la composition d'une histoire de la philosophie n'appartient plus à un philosophe qu'à un autre. C'est dans cette perspective qu'il se charge, dans l'Encyclopédie, de révéler les analogies entre certains principes appartenant à différents systèmes. Or le nombre d'analogies augmente à mesure que s'étend notre connaissance des textes philosophiques. Diderot avait suggéré dans l'article ECLECTISME que les exemples de rencontres des Modernes avec les Anciens seraient « encore plus communs, si nous n'avions perdu aucune des productions de l'antiquité $»^{8}$. Naigeon

7. Panckoucke, «Vocabulaire encyclopédique », EM, second Prospectus, Mathématiques, t. III, Paris, Panckoucke, 1789, p. 49 ; M. Groult (éd), Charles-Joseph Panckoucke, Prospectus et mémoires de l'Encyclopédie méthodique, vol. II, 1789-1792, Paris, Classiques Garnier, 2013, p. 263-264.

8. Diderot, article ECLECTISME (Histoire de la philosophie ancienne et moderne), Enc., V, 273. 
semble avoir senti la justesse de cette hypothèse : non seulement il accorde la plus grande attention à la philosophie ancienne, mais il multiplie le nombre d'extraits de textes philosophiques. On observe cependant qu'il s'écarte de l'éclectisme diderotien. Il critique notamment le fait que Diderot lise tous les philosophes en s'identifiant à eux sans s'en apercevoir. Ainsi Diderot "prêtait quelquefois ses idées » aux Anciens, " et s'appropriait de même les leurs, à-peu-près comme des amis dont les biens sont communs et qui vivent solidaires » (Discours préliminaire, $\mathrm{p}$. VI). On reconnaîtra ici une réécriture du passage de l'article ECLECTISME où Diderot décrit les philosophes éclectiques comme étant « parmi les philosophes ce que sont les souverains sur la surface de la terre, les seuls qui soient restés dans l'état de nature où tout était à tous » $(E n c ., \mathrm{V}, 270)$.

Cette critique de l'éclectisme diderotien révèle que l'enjeu de la réédition du corpus des articles d'histoire de la philosophie dans l'Encyclopédie méthodique est moins de mettre en lumière de nouvelles analogies entre les principes appartenant à la philosophie ancienne ou à la philosophie moderne que d'apprécier à leur juste mesure la nouveauté d'une idée ainsi que l'invention d'un auteur. Naigeon poursuit la recherche des convergences entre les philosophies engagée par Diderot tout en renouvelant non seulement les matériaux mais aussi la méthodologie employée par ce dernier. En s'attachant au vocabulaire philosophique, il met l'accent sur l'historicité des principes. Sa lecture reste néanmoins cohérente avec la contribution de Diderot à l'histoire de la philosophie dans l'Encyclopédie, car, si elle se démarque de l'éclectisme, elle donne plus de relief à l'idée que tous les systèmes philosophiques s'inscrivent dans un même horizon: ils participent d'une philosophie générale dont les éléments ont été donnés par Diderot dans l'Encyclopédie.

Dans l'avertissement pour l'Encyclopédie méthodique publié dans le Mercure de France, Naigeon présente son projet de dictionnaire en annonçant qu'il prévoit « d'étendre ou d'abréger, de refaire même en tout ou en partie » certains articles de philosophie ancienne et moderne de la première Encyclopédie mais qu'il « conservera scrupuleusement, et sans se permettre même le plus léger changement, ceux qui sont le fruit des recherches d'un Philosophe justement célèbre, qui a porté sur tous les objets dont il s'est occupé, des vues également neuves, fines et profondes ». Naigeon marque tout se qui le sépare de J. Brucker, dont l'histoire critique est la principale source de Diderot en matière d'histoire de la philosophie. Il s'engage non seulement à lire les auteurs et à en extraire les matériaux susceptibles de " répandre quelque jour sur la philosophie ancienne et moderne », mais aussi à les lire « dans un autre esprit, et avec d'autres vues que Brucker ». Ainsi est-ce 
autant par la qualité encyclopédique de sa lecture que par la nouveauté des matériaux qu'il introduit que Naigeon se pose comme l'auteur d'une nouvelle histoire de la philosophie. Dans cette toute première version du projet, il présente le futur dictionnaire comme contenant une « Histoire générale et particulière de la Philosophie et des Philosophes anciens et modernes $»^{9}$. La réalisation d'un tel projet suppose de la part de Naigeon une lecture très attentive de la contribution de Diderot à l'histoire de la philosophie dans l'Encyclopédie.

Dans la lettre insérée dans le Second Prospectus de l'Encyclopédie méthodique qu'il adresse à Panckoucke, Naigeon marque cette fois-ci ce qui le distingue de Diderot en expliquant comment les extraits insérés par ce dernier dans les articles d'histoire de la philosophie « ne sont souvent que la traduction de ceux de Brucker $»^{10}$. La différence entre l'Encyclopédie et l'Encyclopédie méthodique, en matière d'histoire de la philosophie, réside non seulement dans les sources, qui diffèrent d'une édition à l'autre, mais aussi dans l'usage de celles-ci : on passe d'un travail de traduction à un travail d'édition de textes philosophiques. Par exemple, le corpus des opuscules leibniziens se présente dans l'article LEIBNITZIANISME de l'Encyclopédie comme une traduction du latin au français d'après J. Brucker, alors que Naigeon rééditera dans l'Encyclopédie méthodique plusieurs opuscules rédigés par Leibniz en langue française. Ce travail éditorial reste néanmoins soumis aux règles de l'écriture encyclopédique en matière d'histoire de la philosophie, Naigeon se situant sous cet aspect en parfaite continuité avec Diderot.

Le fait que Diderot ait lui-même formé le projet d'une seconde édition des articles d'histoire de la philosophie justifie d'ailleurs amplement la contribution de Naigeon à l'Encyclopédie méthodique. Comme l'explique ce dernier, l'édition d'une histoire de la philosophie dans l'Encyclopédie méthodique répond au vœu de Diderot qui « regrettait de n'avoir pas donné à cette partie de l'histoire des progrès de l'esprit humain une attention et des soins qui répondissent à l'importance de l'objet ». Diderot, poursuit Naigeon, « se proposait d'y suppléer dans une seconde édition $»^{11}$, et en avait conçu le plan. Ainsi Naigeon

9. Encyclopédie méthodique, ou par ordre des matières, «XVI. La Philosophie ancienne et moderne, par M. Naigeon », Mercure de France, 8 décembre 1781, p. $119-122$.

10. EM, «Philosophie ancienne et moderne par M. Naigeon», «Lettre de l'auteur à $\mathrm{M}$. Panckoucke, contenant des vues générales sur la manière de traiter l'Histoire critique de la Philosophie [...] », 16 février 1788, Mathématiques, t. III, p. 16 ; éd. M. Groult, o.c., p. 181 ; J. Brucker, Historia critica philosophiae, Leipzig, 1742-1744.

11. «Lettre de l'auteur à M. Panckoucke (...), EM, Mathématiques, t. III, p. 16 ; éd. M. Groult, o.c., p. 180. 
expose-t-il les grandes lignes d'un projet dont J. Brucker a certes fourni de nombreux matériaux à Diderot, mais dont l'esquisse revient à ce dernier.

En présentant ce projet sous l'intitulé « Histoire philosophique de l'entendement humain considéré dans ses différentes périodes $\rangle^{12}$, Naigeon nous renvoie à l'article ECLECTISME de l'Encyclopédie. Diderot y propose d'expliquer les ressemblances entre la philosophie des anciens et des modernes par le "désordre (...) philosophique » décrit par D'Alembert dans le Discours préliminaire (Enc., I, XIV), et emploie à cet effet l'expression "système de déraison " (Enc., V, 273). Certes, Diderot est conscient du fait que toute ressemblance entre les philosophies est singulière et pose comme telle un problème d'interprétation, cependant c'est le caractère récurrent des ressemblances qui l'intéresse. En décrivant "la marche désordonnée et les écarts du Génie poétique, de l'Enthousiasme, de la Métaphysique, et de l'Esprit systématique ", Diderot fait donc l'esquisse de l'histoire de l'entendement humain considéré, comme le dira plus tard Naigeon, dans ses différentes périodes. Enfin, il en déduit une règle générale, à savoir que « les hommes d'un siècle ne diffèrent guère des hommes d'un autre siècle » et « que les mêmes circonstances amènent presque nécessairement les mêmes découvertes" (Enc., V, 273). On reconnaîtra ici la remarque formulée par Fontenelle dans sa Digression sur les anciens et les modernes : " Les siècles », écrit-il, " ne mettent aucune différence naturelle entre les hommes $\gg{ }^{13}$.

S'il est évident que Diderot se réfère à Fontenelle dans l'article ECLECTISME, il est surprenant que Naigeon se contente de citer ce dernier lorsqu'il énonce à son tour les grandes lignes de l'« Histoire philosophique de l'entendement humain» (Discours préliminaire, p.X.). En effet, ce n'est pas à Diderot mais à Fontenelle que renvoie Naigeon lorsqu'il avance des arguments en faveur de l'utilité de l'histoire philosophique de l'entendement humain. Sa lecture de Diderot présente la particularité de se référer aux sources de celui-ci. Ainsi déclare-t-il qu' « on ne peut guère douter de ce que Fontenelle observe quelque part, que l'histoire des folies des hommes ne soit une grande partie du savoir, et que malheureusement plusieurs de nos connaissances

12. « Lettre de l'auteur à M. Panckoucke (...), EM, Mathématiques, t. III, p. 18 ; éd. M. Groult, o.c., p. 184 ; EM, Philosophie ancienne et moderne, Discours préliminaire, t. I, p. X.

13. Fontenelle, Poésies pastorales de M. D. F. avec un Traité sur la nature de l'Eglogue, et une Digression sur les anciens et les modernes, 1688, p. 233. 
ne se réduisent là $\gg^{14}$. L'histoire de l'entendement humain ne dépend ni des climats ni des siècles, bien que certaines circonstances peuvent en infléchir le cours ; c'est dans l'entendement qu'il faut rechercher le principe de sa propre histoire. Naigeon envisage par conséquent de considérer les différentes périodes de l'entendement humain, et décrit celles-ci de la même façon que Diderot tout en renvoyant à une des principales sources de ce dernier, c'est-à-dire à Fontenelle. Nous pensons au passage de l'article ECLECTISME où Diderot écrit que la « première des causes du retardement de l'Éclectisme moderne, est la route que suit naturellement l'esprit humain dans ses progrès, et qui l'occupe invinciblement pendant des siècles entiers à des connaissances qui ont été et qui seront dans tous les temps antérieures à l'étude de la Philosophie » (Enc., V, 284). Diderot reprend ensuite la comparaison faite par Fontenelle entre les progrès de la raison et les âges de l'homme ${ }^{15}$. Ainsi s'exclame-t-il en conclusion : "L'esprit humain a son enfance et sa virilité : plût au ciel qu'il n'eût pas aussi son déclin, sa vieillesse et sa caducité » (Enc., V, 284).

Dans la Digression sur les Anciens et les Modernes, Fontenelle envisage l'histoire des sciences et des arts en remarquant que ceux-ci progressent différemment selon les facultés qu'ils sollicitent. Ainsi, la perfection des sciences dépend de la raison et, si les progrès en sont lents, ils ne connaissent pas de limites ${ }^{16}$. Fort de ce principe, Fontenelle peut se placer du point de vue de la postérité et affirmer qu'il sera aussi absurde d'admirer Descartes dans deux ou trois mille ans qu'il est nuisible à l'époque où il écrit d'admirer Aristote, car les Modernes finiront par devenir les contemporains des Anciens. On comparera ce développement avec le passage de l'Éloge de Leibniz où Fontenelle explique en quel sens l'« Histoire des pensées des hommes ${ }^{17}$ peut être instructive. La matière première de cette histoire provient principalement de la lecture des philosophes. Celle-ci fournit un nombre infini de matériaux à la pensée. Elle indique les écueils à éviter et les routes à suivre. Enfin, elle apprend aux génies qu'il leur arrive de se tromper. Ce passage de l'Éloge de Leibniz inspire à Diderot dans l'article LEIBNITZIANISME une réflexion originale sur l'historicité de toute

14. « Lettre de l'auteur à M. Panckoucke [...], EM, Mathématiques, t. III, p. 18 ; éd. M. Groult, o.c., p. 184 ; EM, Philosophie ancienne et moderne, Discours préliminaire, t. I, p. X ; Fontenelle, Histoire de l'Académie royale des sciences, année 1708, Paris, 1709, p. 110-111.

15. Digression sur les anciens et les modernes, o.c., p. 265-267.

16. Digression sur les anciens et les modernes, o.c., p. 244-245.

17. Fontenelle, «Éloge de M. Leibnitz », Éloges des académiciens, La Haye, 1740, t. I, p. $443-444$. 
découverte scientifique. Il remarque qu'en cherchant à concilier l'aristotélisme avec la philosophie moderne, Leibniz a montré "qu'une erreur surannée est quelquefois le germe d'une vérité nouvelle » (Enc., IX, 371). Naigeon adopte ce principe d'interprétation en lui donnant toute la portée qu'il mérite : il s'attachera, à la suite de Fontenelle et des éditeurs de l'Encyclopédie, à recueillir « tout ce que, dans une longue suite de siècles marqués dans l'histoire par des époques plus ou moins longues de barbarie et de lumière, l'esprit humain a pensé de plus absurde et de plus judicieux, de plus extravagant et de plus raisonnable, de plus conjectural et de plus précis ». Il s'agit bien de considérer l'entendement humain "dans ses accès divers de force et de faiblesse, de raison et de folie » (Discours préliminaire, p. x), étant supposé que l'homme passe nécessairement par une série d'erreurs avant d'arriver à la vérité et que la connaissance des routes conduisant à l'erreur lui est utile afin d'accéder plus librement à celle menant à la vérité.

Ce développement nous renvoie aux premiers paragraphes du Discours préliminaire du dictionnaire de Philosophie ancienne et moderne. Naigeon y développe une lecture inédite du «tableau du renouvellement de la philosophie éclectique » à l'époque moderne ébauché par Diderot dans l'article ECLECTISME. Ce dernier s'attache notamment à définir les différentes causes du retard de la philosophie éclectique en général : la première cause de ce retard, explique-t-il, " est nécessaire, inévitable, et fondée dans la nature des choses", tandis que les autres causes sont « accidentelles et conséquentes à des événements que le temps pouvait ou ne pas amener, ou du moins amener dans des circonstances moins défavorables ». Or Naigeon ne retiendra de cette explication que la première cause, à savoir celle définie par Diderot comme nécessaire, ce qui s'avère tout à fait cohérent avec l'idée formulée par Fontenelle et par les encyclopédistes avant que Naigeon ne la fasse sienne, idée selon laquelle c'est dans l'entendement qu'il faut rechercher le principe d'une histoire philosophique.

Il reste à déterminer dans quelle mesure la lecture de Naigeon restitue la pensée de Diderot. À lire le texte de l'article ECLECTISME, il apparaît qu'il comporte plusieurs éléments pouvant effectivement conduire à envisager avec Naigeon que les causes ayant retardées les progrès de la philosophie sont absolument toutes nécessaires. Il semble en effet que l'histoire de l'entendement humain n'obéisse pas uniquement à une nécessité propre à l'esprit humain mais soit aussi déterminée par des circonstances ou des événements ne relevant pas de la seule nécessité naturelle. Cette possibilité de lecture est confirmée par le fait que Diderot précise qu'il choisit de suivre la façon commune de penser en distinguant ainsi des causes nécessaires et accidentelles : « Je me conforme ", écrit-il, " dans cette distinction à la manière commune 
d'envisager les choses, et je fais abstraction d'un système qui n'entraînerait que trop facilement un homme qui réfléchit avec profondeur et précision, à croire que tous les événements dont je vais parler, sont également nécessaires » (Enc., V, 283-284). Les premiers paragraphes du Discours préliminaire du dictionnaire de Philosophie ancienne et moderne peuvent par conséquent être considérés comme une explication inédite du tableau du renouvellement de la philosophie éclectique ébauché par Diderot dans l'Encyclopédie. Cette explication, loin de se conformer à la manière commune de penser, révèle l'adhésion de Diderot à l'hypothèse de la nécessité et restitue la pensée de ce dernier concernant l'histoire de la philosophie en général.

Cette nouvelle ébauche de l'histoire de la philosophie s'accompagne dans le Discours préliminaire du dictionnaire de Philosophie ancienne et moderne d'une réécriture du texte diderotien où l'on remarque la présence d'idées philosophiques centrales pour la compréhension de la pensée diderotienne. Naigeon emprunte à Diderot l'idée $\mathrm{d}^{\prime}$ « inquiétude automate $»^{18}$ en l'attribuant non plus aux molécules organiques mais aux hommes de génies. On comparera aux Pensées sur l'interprétation de la nature le passage suivant du Discours préliminaire $d u$ dictionnaire de Philosophie ancienne et moderne : "les hommes doués de quelque aptitude extraordinaire pour les sciences ou pour les arts [...] ont tous, plus ou moins, cette espèce d'inquiétude automate qu'on remarque dans les animaux quelques moments avant leur sommeil, et qui les porte à s'agiter en tout sens, à changer sans cesse de position, jusqu'à ce qu'ils rencontrent celle qui est la plus commode pour eux, et qui finissent toujours par la trouver » (Discours préliminaire, t. I, p. III-IV). La nécessité de s'exercer, pour l'entendement humain, prévaut sur l'objet qui l'occupe ainsi que sur les circonstances de son exercice. Ainsi, « les hommes doués de quelque aptitude extraordinaire pour les sciences ou pour les arts (...) font presque toujours la chose à laquelle ils sont particulièrement propres, et vers laquelle ils se sentent le plus fortement entraînés » (Discours préliminaire, t. I, p. III). Naigeon poursuit en décrivant le philosophe et l'érudit comme « deux espèces d'automates montés pour une certaine suite de mouvements divers, deux machines nécessairement disposées, organisées, l'une pour avoir beaucoup d'esprit, de jugement et d'idées, et pour faire de la raison ; l'autre, pour retenir, par exemple, à-peu-près tous les mots d'une langue morte et leurs radicaux, pour savoir dans quel sens chacun de ces mots est employé dans tel et tel auteur ancien, et le passage original ou de quelque vieux scholiaste où cette expression se trouve » (Discours préliminaire, t. I, p. v). Cette reformulation 
de l'idée diderotienne d'inquiétude permet à Naigeon d'expliquer que l'érudition et la critique sont des instruments dont l'usage n'est pas naturel au philosophe : ainsi Diderot, écrit-il, était « absolument incapable de cette patience, de cette exactitude si nécessaire dans l'examen des faits ; et cette attention scrupuleuse que dans ces recherches et dans ces discussions arides il faut sans cesse donner à une foule de petits objets, qui ont néanmoins leur utilité, était surtout au-dessus de ses forces ». Pourtant, la composition d'une histoire de la philosophie dans l'Encyclopédie exigeait, comme le précise Naigeon, de recourir à l'érudition et à la critique autant qu'à la philosophie.

Toujours dans le Discours préliminaire, Naigeon met en évidence la fonction encyclopédique de l'histoire de la philosophie en affirmant qu' « il est peu de parties dans l'Encyclopédie d'une utilité plus générale, plus constante, et qui par la profondeur des matières qu'elle embrasse, par le nombre et la nature des idées qu'elle réveille dans l'esprit, et qui sont quelquefois à une grande distance des premières, offre un champ plus vaste à la méditation » (Discours préliminaire, p. VI). Il revient à Diderot d'avoir été, avec Bacon, à l'origine de la naissance de l'histoire de la philosophie envisagée dans une perspective encyclopédique. Naigeon cite un passage de De Dignitate et Augmentis scientiarum dans lequel ce dernier forme le plan d'une « histoire des opinions des anciens philosophes $\rangle^{19}$. Il en présente le projet en notant que celui-ci a le mérite de reconnaître l'histoire de la philosophie comme constituant une branche des connaissances humaines à part entière. De plus, la méthode qu'il préconise rejoint l'approche encyclopédique des systèmes de la connaissance humaine. Bacon recommande en effet d'exposer à part chaque philosophie, d'en lier les différents dogmes, de telle sorte que ceux-ci s'éclaircissent réciproquement et que chaque philosophie forme un tout. Bacon aurait ainsi découvert, selon les termes de Naigeon, « le point de vue d'où il fallait considérer chaque objet pour en saisir l'ensemble, et le pénétrer, pour ainsi dire, tout entier d'un coup d'œil $»^{20}$. On reconnaît ici la terminologie des éditeurs de l'Encyclopédie lorsqu'ils forment le projet de représenter l'enchaînement des sciences en composant le système le plus lié possible. C'est par la généralité de son objet que l'histoire de la philosophie tend ainsi à se confondre avec le projet encyclopédique. Naigeon pourra de ce point de vue critiquer la méthodologie employée par Dutens dans son ouvrage Recherches sur l'origine des découver-

19. EM, Philosophie ancienne et moderne, Discours préliminaire, t. I, p. I ; Bacon, De Dignitate et Augmentis scientiarum, livre III, chapitre IV, éd. Paris, 1624, p. 181.

20. Philosophie ancienne et moderne, Discours préliminaire, t. I, p. I. 
tes ${ }^{21}$, méthodologie consistant à rechercher l'origine d'une idée dans une idée antérieure. Le défaut de cette méthode est de considérer les idées sans en comprendre vraiment la liaison et sans tenir compte du fait qu'elles appartiennent à telle ou telle philosophie. Or, comme le remarque Naigeon, en reformulant une réflexion de Diderot, une idée « ne se trouve pas isolée, solitaire dans la tête de l'inventeur; elle n'y est pas arrivée brusquement, et pour ainsi dire, à son insu; il a au moins une partie de celles auxquelles cette idée correspond, et qui ont pu l'y conduire » (Discours préliminaire, p. XXI).

Si le projet baconien retient l'attention de Naigeon, c'est qu'il accorde une place à l'histoire de la philosophie dans l'enchaînement des sciences et conçoit celle-ci comme offrant l'accès à un point de vue général. Cependant, Naigeon ne puise pas aux sources de l'Encyclopédie sans conserver la terminologie des éditeurs de l'ouvrage. Nous pensons à la notion de " point de vue $»^{22}$ définie par D'Alembert dans le Discours préliminaire puis par Diderot dans l'article ENCYCLOPÉDIE, ce dernier recommandant par exemple aux auteurs des articles de saisir « le point de vue » $(E n c ., \mathrm{V}, 642)$ sous lequel considérer la chose dont ils traitent. Naigeon emploiera à son tour la notion de point de vue à des fins critiques en observant que si J. Brucker « avait envisagé son sujet sous son vrai point de vue, et dans tous ses rapports, il aurait fait un beau livre » (Discours préliminaire, p. Ix).

Ainsi l'histoire de la philosophie pourrait participer à l'enchaînement des sciences par la généralité et l'étendue de sa matière, ou encore par la mise en ordre des idées qu'elle effectue. Il est d'ailleurs significatif que Naigeon emploie le terme d' " homme en général » lorsqu'il envisage l'histoire philosophique de l'entendement humain, ou encore qu'il parle de "science générale des anciens » (Discours préliminaire, p. x) afin de désigner la philosophie ancienne. On comparera cet emploi avec celui que fait Diderot dans l'article ENCYCLOPÉDIE en désignant par le terme de "science générale » la " science de l'homme en général»(Enc., V, 635 et 640). Ainsi Naigeon est-il amené à redéfinir les moyens d'élever les connaissances humaines à la plus grande généralité, y compris en matière d'histoire de la philosophie. Il décrit comment le philosophe, tout en travaillant sur les mêmes matériaux que l'érudit, à savoir sur les faits, parvient à en saisir la logique, c'est-à-dire à les ordonner, à en déduire des vérités et, finalement, à accéder à un degré d'universalité toujours plus élevé. Ce qui se dessine 1776.

21. L. Dutens, Recherches sur l'origine des découvertes attribuées aux modernes,

22. D'Alembert, Discours préliminaire, Enc, I, p. XIV. 
ici est la possibilité d'une " histoire de la philosophie en général $»^{23}, \mathrm{ou}$ encore d'une philosophie dont le degré d'universalité serait plus grand que celui de toute philosophie particulière. De fait, l'esquisse de cette histoire philosophique prend progressivement forme au cours de la rédaction des trois volumes du dictionnaire de Philosophie ancienne et moderne.

Dès le Discours préliminaire, Naigeon aborde la question du progrès de la langue philosophique. «Ce qui rend la Physique et la Métaphysique des anciens si vague, si obscure, si difficile à entendre ", écrit-il, "c'est qu'il n'avaient pas, si l'on peut s'exprimer ainsi, la langue de leurs idées » (Discours préliminaire, p. XIV). Ce passage du Discours préliminaire se situe dans le prolongement des réflexions formulées par Diderot dans l'article ENCYCLOPÉDIE, ce dernier énonçant que « la langue est une image rigoureuse et fidèle de l'exercice de la raison » (Enc., V, 638). Ainsi, la connaissance de la langue permettrait d' " éclaircir l'histoire des progrès de l'esprit humain dans les siècles passés » (Enc., V, 637). Naigeon portera la plus grande attention à cette dernière remarque, à tel point qu'il verra dans les articles de grammaire rédigés par Diderot pour l'Encyclopédie des articles d'histoire de la philosophie.

L'article PRODUCTION, désigné dans le dictionnaire de Philosophie ancienne et moderne comme un article d'histoire de la philosophie, nous fournira un exemple de la lecture du texte encyclopédique diderotien par Naigeon. Précisons qu'il s'agissait dans l'Encyclopédie d'un article de grammaire ${ }^{24}$. Naigeon y explique en introduction comment, dans la première Encyclopédie, l'ordre alphabétique prévalait sur celui des matières, ce qui avait conduit les éditeurs de l'ouvrage à présenter les articles d'« histoire de philosophie en général » comme s'il s'agissait de "simples articles de mots » ou de " grammaire philosophique $»^{25}$. Or ces articles relèvent de l'histoire de la philosophie à partir du moment où ils sont considérés dans l'Encyclopédie méthodique par ordre de matières. Naigeon explique encore qu'ils contiennent des matériaux que Diderot a volontairement dispersés dans l'ouvrage, suite à la censure à laquelle celui-ci faisait l'objet. Ainsi Naigeon

23. EM, Philosophie ancienne et moderne, article PRODUCTION (Histoire de la philosophie), t. III, p. 466.

24. Diderot, article PRODUCTION (Grammaire), Enc., XIII, 424.

25. EM, article PRODUCTION, t. III, p. 466. Naigeon remplace généralement le désignant « Grammaire » par l'expression « Grammaire philosophique ». Sur la grammaire philosophique dans l'Encyclopédie, voir M. Leca-Tsiomis, Écrire l'Encyclopédie. Diderot, de l'usage des dictionnaires à la grammaire philosophique, Oxford, Voltaire Foundation, (1999) 2007. 
procède, par sa lecture des articles de grammaire rédigés par Diderot pour l'Encyclopédie, à une forme de restitution dont l'objet n'est pas le texte, celui-ci restant le même, mais le projet philosophique diderotien: les articles de grammaire présentés par Naigeon comme des articles d'histoire de la philosophie contiennent, écrit celui-ci en s'inspirant d'Horace, «les membres dispersés d'un philosophe $»^{26}$. Les termes de cette introduction sont ceux employés par Naigeon dans le Discours préliminaire. Ce dernier écrivait alors que « la plupart des matériaux qu'il [Diderot] avait recueillis dans le silence de l'étude et de la méditation, et qui, employés avec discernement par d'aussi habiles mains, auraient été si utiles pour perfectionner l'entendement humain, et pour mettre dans la langue philosophique cette exactitude et cette précision sans lesquelles on n'éclaircit rien, se trouvent épars, isolés, perdus dans des articles où personne jusqu'à présent ne les a découverts, où personne même ne les suppose, ne les cherche, et où Diderot semble même les avoir déposés, moins comme des vérités positives, déjà constatées par l'expérience, et pour ainsi dire jugées et senties, que comme des espèces de germes qui attendent leur développement et leur fécondation du progrès des lumières et de l'irradiation des esprits " (t. III, p. 466). Enfin, à la fin de l'article PRODUCTION, Naigeon renvoie le lecteur à l'article DIDEROT (Philosophie de), et plus précisément au passage où il expose le projet diderotien de « dictionnaire universel et philosophique de la langue ». Il explique dans ce passage comment Diderot «a dispersé dans l'Encyclopédie un grand nombre de matériaux qui devaient servir un jour à la composition de ce vocabulaire par lequel il avait résolu de terminer sa carrière littéraire $»^{27}$. Cette description reformule de façon résumée le projet diderotien de vocabulaire universel présenté au début de l'article ENCYCLOPÉDIE de l'Encyclopédie, Naigeon précisant qu'un tel projet repose sur l'hypothèse d'un rapport entre la connaissance de la langue et le projet encyclopédique. Diderot insiste effectivement sur ce rapport en déclarant : « C'est de la perfection de l'idiome que dépendent et l'exactitude dans les sciences rigoureuses, et le goût dans les Beaux-Arts, (...)» (Enc., V, 635-638). Naigeon énonce pour sa part que «la perfection de l'entendement humain tient plus qu'on ne croit à celle de la grammaire $»^{28}$, ce qui n'est pas non plus sans faire écho à l'idée que la science des signes et des

26. EM, article PRODUCTION, t. III, p. 466. On lit en note : «Invenias etiam disjecti membra philosophi ». Dans les Satires, Horace parlait du poète.

27. EM, article DIDEROT (philosophie de) (Histoire de la philosophie moderne), Histoire ancienne et moderne, t. II, p. 219.

28. EM, article DIDEROT (philosophie de), Histoire ancienne et moderne, t. II, p. 220 ; article ENCYCLOPÉDIE, Enc., V, 637. 
sons a plus de liaison avec la science des choses qu'on ne le croit et pourrait servir à éclaircir l'histoire des progrès de l'esprit humain.

Nous mentionnerons un dernier exemple d'article de grammaire présenté par Naigeon comme un article d'histoire de la philosophie et témoignant de la façon dont il interprète la contribution de Diderot à l'histoire de la philosophie. Il s'agit de l'article NATURE (INTEMPÉRIE DE LA) présenté par le désignant "Histoire de la philosophie moderne ». Il s'agit dans l'Encyclopédie de l'article de grammaire INTEMPÉRIE. Le texte de l'article pour être court n'en est pas moins philosophique. Naigeon n'en présente qu'un extrait, ne conservant finalement de l'article que sa matière philosophique. Le choix d'en modifier l'entrée, en mettant en valeur l'idée de nature, s'explique par la matière même de l'article et par le principe qui y est énoncé, à savoir que " l'ordre des choses est nécessaire ${ }^{29}$. Enfin, Naigeon insère un renvoi final au paragraphe « Sur les causes finales » de l'article DIDEROT (philosophie de). En extrayant de l'Encyclopédie les matériaux utiles à la constitution d'une histoire de la philosophie, Naigeon présente une lecture philosophique de textes qui n'y étaient pas présentés comme relevant de l'histoire de la philosophie. Ainsi le paragraphe «Sur les causes finales » est composé de deux articles de grammaire rédigés par Diderot pour l'Encyclopédie : l'article LAIDEUR et l'article HARMO$\mathrm{NIE}^{30}$. Naigeon met donc à jour, notamment au moyen de renvois, les matériaux épars du vocabulaire universel dont Diderot avait le projet.

Ce n'est que tardivement, semble-t-il, que Naigeon présente ainsi des échantillons du projet philosophique diderotien à partir d'éléments textuels initialement insérés à l'Encyclopédie, puisqu'il réserve ces échantillons pour le troisième volume du dictionnaire de Philosophie ancienne et moderne. Rappelons cependant qu'il prévoyait dans la première version de son projet de traiter de l'histoire générale et particulière de la philosophie et qu'il annonçait alors le titre suivant : « Histoire générale et particulière de la Philosophie et des Philosophes anciens et modernes $»^{31}$. En présentant une sélection d'articles de grammaire dont la matière relève de l'histoire de la philosophie, Naigeon revient finalement à sa première idée et reconstitue en partie le

29. EM, article NATURE (INTEMPÉRIE DE LA), Histoire ancienne et moderne, t. III, p. 350 ; Diderot, article INTEMPÉRIE (Grammaire), Enc., VIII, 806-807.

30. EM, article DIDEROT (philosophie de), «Sur les causes finales », Histoire ancienne et moderne, t. II, p. 203-204 ; Diderot, article LAIDEUR (Grammaire et morale), Enc., IX, 176 ; article HARMONIE (Grammaire), Enc., VIII, 50.

31. Mercure de France, p. 119. 
projet diderotien d'un « dictionnaire universel et philosophique de la langue $»^{32}$.

La lecture de Diderot engagée par Naigeon nous invite à relire l'Encyclopédie en étant sensible à la place de l'histoire de la philosophie dans l'enchaînement des sciences. La constitution de cette histoire au cours de la rédaction de l'ouvrage apparaît indissociable du projet encyclopédique diderotien, celui de composer un vocabulaire universel et philosophique. Que Diderot en ait dispersé les éléments dans l'Encyclopédie afin de les transmettre à la postérité ne fait que donner plus de sens à la lecture de Naigeon dont témoigne l'édition du dictionnaire de Philosophie ancienne et moderne de l'Encyclopédie méthodique, car il revient à ce dernier d'avoir mis en ordre les matériaux d'une véritable histoire de la philosophie.

Université Montpellier III CRISES-E.A. 4424

32. EM, article DIDEROT (philosophie de), Histoire ancienne et moderne, t. II, p. 219. 